\title{
Crossfield Sensitivity in AMR Sensors
}

\author{
Pavel Ripka, Michal Janošek, and Mattia Butta \\ Department of Measurement, Faculty of Electrical Engineering, Czech Technical University in Prague, \\ 16627 Praha 6, Czech Republic
}

\begin{abstract}
We discuss the origin of the crossfield sensitivity of AMR sensors, the way how this error may influence the performance of an AMR compass and methods for its correction. Finally, we confirm the simple formulas experimentally. Crossfield may cause compass error up to 2.6 deg., depending on the compass orientation. The most effective way to suppress the crossfield error is using magnetic feedback, however this is not always possible. We suggest a method of processing of the SET/RESET sensor outputs which is more efficient than the usual averaging.
\end{abstract}

Index Terms-AMR, crossfield sensitivity, magnetic sensors, magnetoresistance.

\section{INTRODUCTION}

C ROSSFIELD effect (or crossfield error) is defined as an unwanted non-linear sensitivity to small fields perpendicular to the sensing direction [1]. This effect can be found in any sensor containing ferromagnetic material. While in fluxgate this error can be minimized by proper design, for AMR sensors the crossfield sensitivity is their inherent property, as it is caused by the basic sensor principle.

If the crossfield is large, it causes irreversible changes: in case of AMR, the single domain state is broken and the anisotropy dispersion occurs [2]. We do not consider this situation in this paper.

We also do not speak about the linear part of the crossfield sensitivity: it can be easily corrected by rotating the sensor. Nonlinear dependence effectively means that one cannot find a universal "insensitive" direction as such direction is field-dependent. The first widely published technical description of this problem was made by Pant and Caruso [3]. The problem is serious: for a sensitive AMR used in magnetic compass a maximum crossfield error in the Earth's field is $1100 \mathrm{nT}$, which may cause an azimuth error of 2 degrees [4]. Some manufacturers reacted to this challenge by changing the design. By increasing anisotropy field they reduced the crossfield error, but also decreased the sensor sensitivity and thus degraded the noise performance. In this paper we will show that crossfield error is easy to understand and we also discuss methods on how to suppress or eliminate this error without compromising the other parameters of AMR sensor.

The simplified formula for the sensor output is [3]:

$$
V_{1} \approx \frac{H_{y}}{H_{x}+H_{0}}
$$

where $H_{\mathrm{y}}$ is the measured field, $H_{\mathrm{x}}$ is the crossfield, and $H_{0}$ is the anisotropy field.

This formula shows that the effect is not present if $H_{\mathrm{y}}=0$.

We will show how (1) is derived and what are the simplifications leading to this formula. But before that we will show the influence of the crossfield error on AMR compass.

Manuscript received March 06, 2009. Current version published September 18, 2009. Corresponding author: P. Ripka (e-mail: ripka@ fel.cvut.cz).

Color versions of one or more of the figures in this paper are available online at http://ieeexplore.iee.org.

Digital Object Identifier 10.1109/TMAG.2009.2022051

\section{Compass ERror CAUSED By CROSSFIELD}

Small and low-cost magnetic compasses are required for cars, ships and mobile devices. Digitally gimbaled compass is using three-axial magnetic sensor and information about actual roll and pitch of the device to calculate azimuth in every position. Precise compass devices usually use classical fluxgate sensors, which are quite large, expensive and power consuming. There are many commercially available compass systems with AMR sensors and inclinometers, but these systems usually have azimuth error of a few degrees. More sophisticated AMR compasses have integrated compensation of offset and sensitivities of individual sensors as well as their angular misalignment. As there are usually six sensors (three magnetic sensors and three accelerometers) in the system, the calibration and error correction algorithm is rather complicated [5]. We suppose that the mentioned errors are compensated and we concentrate on the effect of the crossfield error.

In our application example we use Honeywell HMC 1001 sensor with $H_{0}=1000 \mathrm{~A} / \mathrm{m}$ (i.e., $\mathrm{B}_{0}=800 \mu \mathrm{T}$ ). We can estimate the influence of crossfield error using (1).

If we use horizontally aligned two-axial compass at equator, where the horizontal component of the Earth's field is approx. $50 \mu \mathrm{T}$, the crossfield causes maximum azimuth error of $2.4 \mathrm{deg}$. In arbitrary position this error can reach 2.5 deg [4], [6].

\section{The ORIGIN OF THE SimplifIEd FormULA}

We will try to review how (1) was derived in order to find out which simplifications were made.

Let us suppose an ideal single-domain magnetically soft AMR strip having uniaxial anisotropy with easy direction $\mathrm{x}$ and effective field of $H_{0}$. We assume only coherent rotation of magnetization.

Let us consider a crossfield $H_{\mathrm{x}}$ present simultaneously to the measured field $H_{\mathrm{y}}$. For the total energy density of a single domain of anisotropic material in the magnetic field $\mathrm{H}$ we may write

$$
E=E_{A}+E_{H}=\frac{1}{2} \mu_{0} M_{S} H_{0} \sin ^{2} \varphi-\mu_{0} M_{S} H \cos \alpha
$$

where $M_{s}$ is the saturation magnetization, $\alpha$ is an angle between the magnetization $\mathrm{M}_{\mathrm{s}}$ and external field $H=\left(H_{\mathrm{x}}, H_{\mathrm{y}}\right)$ and $\varphi$ is an angle between the magnetization $\mathrm{M}_{\mathrm{s}}$ and easy direction $\mathrm{x}$. 
In order to find the energy minimum we solve the equation $\mathrm{dE} / \mathrm{d} \varphi$ and if the external field $\mathrm{H}$ is much smaller than the anisotropy field: $\left|H_{\mathrm{x}}\right|,\left|H_{\mathrm{y}}\right| \ll\left|H_{0}\right|$ we will find [2]

$$
\sin \varphi=\frac{H_{y}}{H_{x}+H_{0}} .
$$

The resistance in $\mathrm{x}$ direction is calculated, e.g., in [2]

$R\left(H_{Y}\right)=R_{0}+\Delta R\left[1-\left(\frac{H_{Y}}{H_{x}+H_{0}}\right)^{2}\right]=R_{0}+\Delta R \cos ^{2} \varphi$.

Using Barber poles the resistance equation becomes

$$
R=R_{0}+\Delta R \cos ^{2}\left(\varphi+45^{\circ}\right)
$$

from that we will derive the following formula for the strip resistance:

$$
R=R_{0}^{\prime}+\Delta R \frac{H_{Y}}{H_{x}+H_{0}} \sqrt{1-\left(\frac{H_{y}}{H_{x}+H_{0}}\right)^{2}}
$$

where

$$
R_{0}^{\prime}=R_{0}+\frac{\Delta R}{2} .
$$

For $\left|H_{\mathrm{x}}\right|,\left|H_{\mathrm{y}}\right| \ll\left|H_{0}\right|$ we finally arrive to

$$
R=R_{0}^{\prime}+\Delta R \frac{H_{Y}}{H_{0}} \quad V_{1} \approx \frac{H_{y}}{H_{x}+H_{0}} .
$$

\section{Methods to Eliminate the CRossfield Sensitivity}

\section{A. Feedback Compensation}

From (1) it is clear that there is no response to crossfield $H_{\mathrm{x}}$ when the measured field $H_{\mathrm{y}}$ is zero. That is why the crossfield sensitivity is erased by feedback compensation of the measured field. The limitation of this approach is the homogeneity of the compensation field, which is questionable for flat compensation coil used in case of integrated sensors. However practically achieved linearity of the feedback-compensated KMZ51 was $0.1 \%$, which is sufficient for most applications [7].

Other drawbacks of compensation include increased power consumption and decreased bandwidth.

Ideal compensation is to completely remove the total field. For 3-axial magnetometers this can be achieved by using external 3-axial compensation coil [8], however such solution is very expensive.

\section{B. Increasing $H_{0}$}

We already mentioned that increasing $H_{0}$ improves the linearity in uncompensated mode, and reduces crossfield effect, but decreases sensitivity. $H_{0}$ is an effective anisotropy field, which is a result of both shape anisotropy and induced anisotropy. Thus we can change $H_{0}$ by changing the strip thickness or by magnetic field annealing. The price we pay for this solution is an increase of the sensor noise (in the magnetic field units) which is proportional to the decrease of the sensitivity. Thus in general this solution is effective only for low-end sensors.

\section{Numerical Correction in Multi-Axial Systems}

From the readings of two perpendicular sensors which measure $H_{\mathrm{x}}$ and $H_{\mathrm{y}}$ we can calculate corrected field values supposing that we know the value of $H_{0}$. The equation has no analytical solution, but it can easily be solved numerically. The procedure is in detail described in [3]. The only problem is the knowledge of $H_{0}$. It is not easy to measure it, but fortunately the sensitivity to this parameter is not critical [4].

\section{Crossfield Suppression by Flipping and Averaging}

After flipping (i.e. reversing the remanent magnetization of the AMR strip) the output voltage is changed from $V_{1}$ to $V_{2}$.

$$
V_{2} \approx \frac{H_{y}}{H_{x}-H_{0}} .
$$

Standard technique used in AMR magnetometers is to sample $\mathrm{V}_{1}$ and $\mathrm{V}_{2}$ and subtract them still in the analog form. The resulting voltage $\mathrm{V}$ is then

$$
V \approx \frac{H_{y}}{H_{x}+H_{0}}-\frac{H_{y}}{H_{x}-H_{0}}=\frac{-2 H_{y} H_{0}}{H_{x}^{2}-H_{0}^{2}} .
$$

The sensitivity of $\mathrm{V}$ to the crossfield $H_{\mathrm{x}}$ is largely reduced, as $H_{\mathrm{x}}$ is typically much lower than $H_{0}$.

Using this technique, the residual error in our example (HMC 1001 in $\mathrm{B}=50 \mu \mathrm{T}$ for the measured field $\mathrm{B}_{\mathrm{y}}$ of $20 \mu \mathrm{T}$ ) was reduced from 1000 to $100 \mathrm{nT}$ [6].

\section{E. Separate Processing of $V_{1}$ and $V_{2}$}

We proposed a simple original method to directly calculate both $H_{\mathrm{y}}$ and $H_{\mathrm{x}}$ (if we know $H_{0}$ ) [9]:

$$
\begin{aligned}
& \text { for } \mathrm{V}_{1} \neq \mathrm{V}_{2}: H_{x} \approx H_{0} \frac{\left(V_{1}+V_{2}\right)}{\left(V_{1}-V_{2}\right)}, H_{y} \approx \frac{2 H_{0}\left(V_{1} \cdot V_{2}\right)}{\left(V_{2}-V_{1}\right)} \\
& \text { for } \mathrm{V}_{1}=\mathrm{V}_{2}: H_{\mathrm{y}}=0 .
\end{aligned}
$$

Using this method the crossfield effect is suppressed much more effectively. The uncertainty of estimation of both $H_{\mathrm{y}}$ and $H_{\mathrm{x}}$ is not the same. For practical values of the anisotropy field $H_{0}$ the sensitivity to $H_{\mathrm{y}}$ is much higher than sensitivity to $H_{\mathrm{x}}$. The uncertainty of $H_{\mathrm{x}}$ caused by the magnetic noise depends on $H_{\mathrm{y}}$. In the best case the $H_{\mathrm{x}}$ noise for HMC 1001 is 16-times higher than noise for $H_{\mathrm{y}}$-approximatelly $80 \mathrm{nT}$ p-p. In the worst case we cannot measure $H_{\mathrm{x}}$ when $H_{\mathrm{y}}$ is zero $\left(\mathrm{V}_{1}=\right.$ $-\mathrm{V}_{2}$ ). However, this novel method can be used for simple compass applications and also in two- and three-axial systems to effectively correct for the crossfield error.

This method can be used only for AMR sensors with medium or low value of $H_{0}$, such as Honeywell HMC $1001\left(H_{0}=\right.$ $0.8 \mathrm{mT}$ ) and it is limited to the environment with low magnetic noise.

\section{F. Flipping in Multiaxial Systems}

Flipping can also be used for numerical correction in multiaxial sensor systems. Pant and Caruso have shown that flipping and averaging $\mathrm{V}_{1}$ and $\mathrm{V}_{2}$ in two-axial or triaxial system leads to much faster iterations during the finding of $H_{\mathrm{y}}$ [3]. Also this 

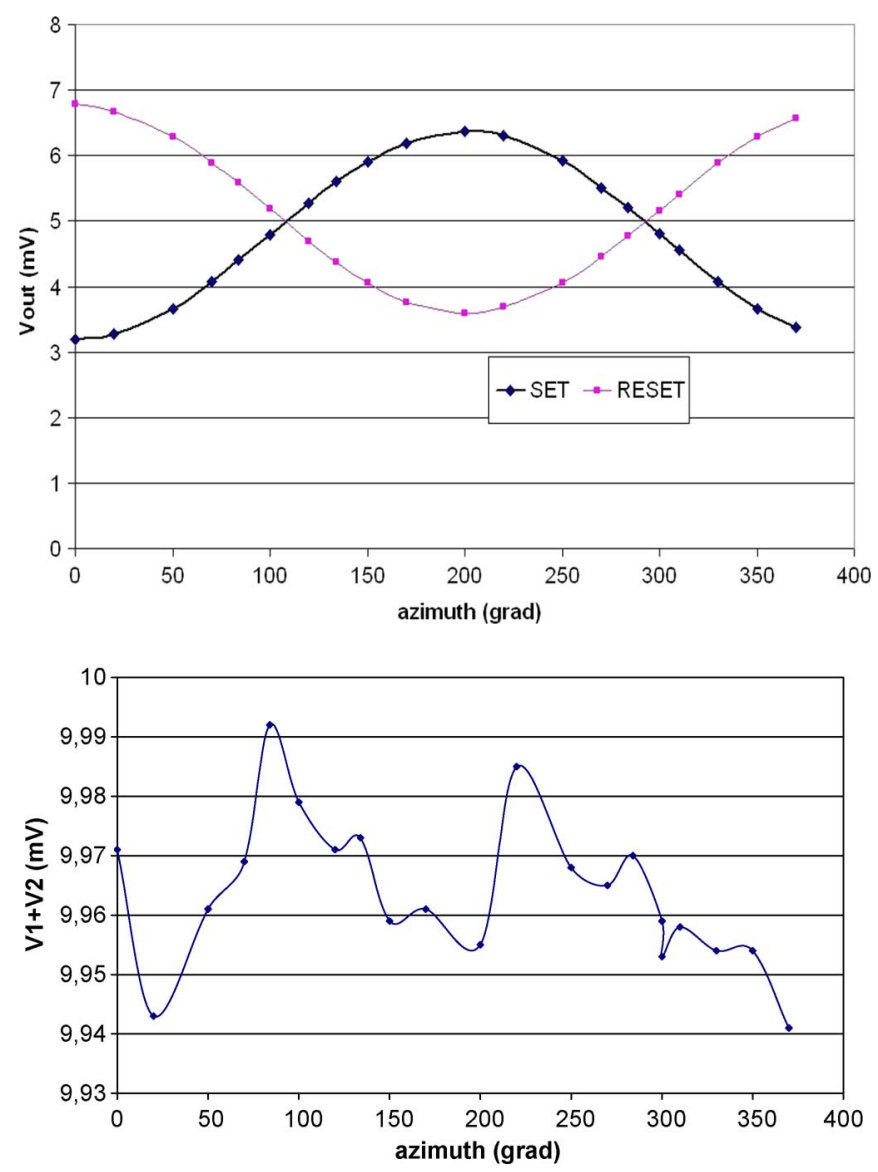

Fig. 1. (a) KMZ 51 sensor $V_{1}$ (SET) and $V_{2}$ (RESET) outputs for rotation in the horizontal plane. (b) KMZ51- $\mathrm{V}_{1}+\mathrm{V}_{2}$ as a function of azimuth.

method requires complex numerical processing and knowledge of $H_{0}$. However, if we do not average but process $\mathrm{V}_{1}$ and $\mathrm{V}_{2}$ for each sensor, we have 6 equations and we need no apriori information about $H_{0}$.

\section{G. Using Redundant Sensors}

We can use 6 sensors in 3-axial AMR magnetometer: XY, $\mathrm{YX}, \mathrm{XZ}, \mathrm{ZX}, \mathrm{YZ}, \mathrm{ZY}$. The first letter denotes the sensing direction, the second letter denotes the crossfield direction. Two letters define the plane in which the sensor chip is mounted. Even without flipping we obtain 6 equations which allow us to compensate for the crossfield error without the knowledge of $H_{0}$. When flipping we can increase the number of equation to 12 . Increasing the number of sensors also increases the complexity of the mentioned scheme for the compensation of angular misalignments.

\section{EXPERIMENTAL VERIFICATION}

Fig. 1(a) shows the output voltages $V_{1}$ and $V_{2}$ as a function of azimuth, measured for Philips KMZ 51 AMR sensor which is used as one axis in magnetic compass. If $\mathrm{V}_{1}-\mathrm{V}_{2}$ value is used, the deviation from sinewave caused by the crossfield effect is practically invisible. However it may cause up to $1 \mathrm{grad}$ compass error. $V_{1}+V_{2}$ value is normally considered to be a sensor offset, which is partly suppressed by flipping procedure.
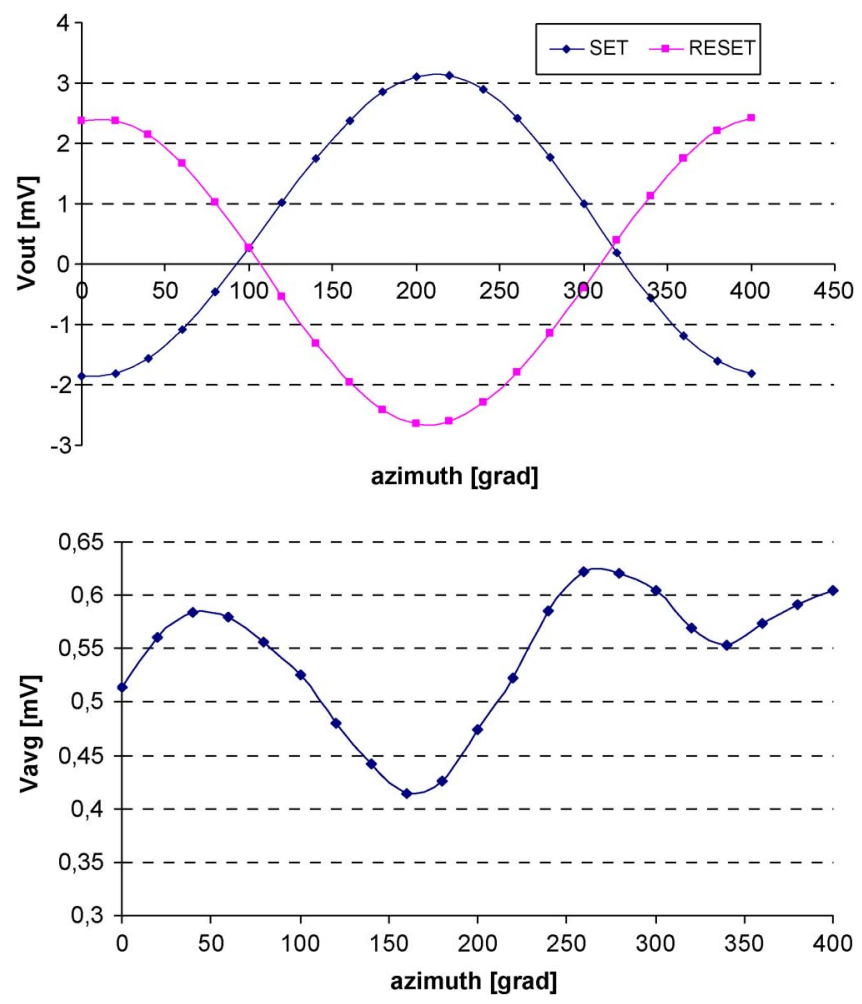

Fig. 2. (a) HMC $1001-V_{1}$ (SET) and $V_{2}$ (RESET) outputs for rotation in the horizontal plane. (b) HMC 1001: $\mathrm{V}_{\mathrm{avg}}=\mathrm{V}_{1}+\mathrm{V}_{2}$ as a function of azimuth.

However, if we measure this value as a function of azimuth for a compass sensor rotating in the Earth's horizontal field of 20000 nT (Fig. 2(b)), we observe a double-peak waveform confirming the simplified formulae (1) and (8). The observed p-p variation of $50 \mu \mathrm{V}$ corresponds to $274 \mathrm{nT}$. Using the simplified formulae this variation gives a rough estimate of $H_{0}=3.2 \mathrm{mT}$ for this sensor.

Fig. 2(a) shows $\mathrm{V}_{1}$ (SET) and $\mathrm{V}_{2}$ (RESET) outputs of Honeywell HMC 1001 sensor (bridge current $6 \mathrm{~mA}$ ) rotated in azimuth. Together with the $\mathrm{V}_{1}+\mathrm{V}_{2}$ function in Fig. 2(b), it indicates smaller value of $H_{0}$ than in KMZ51-this corresponds well with $H_{0}=0.8 \mathrm{mT}$ measured in [4].

In Fig. 3(a), the cross-axis direction of HMC1001 is oriented upwards, so that $\mathrm{Hx}$ is in the rotation axis and therefore constant, $\mathrm{Hx}=40 \mu \mathrm{T}$. In agreement with derived equations the $\mathrm{V}_{1}$ and $\mathrm{V}_{2}$ responses are sinewaves with only slightly different amplitudes. $V_{\text {diff }}=V_{1}-V_{2}$ in this case is close to sinewave (Fig. 3(b)), according to (9). Here we can conclude that the design of HMC 1001 is on the limit of linear AMR sensors: $H_{0}$ is only 16-times higher than the Earth's field.

\section{CONCLUSION}

In this paper we analyzed the crossfield sensitivity of barberpole AMR sensor. Crossfield sensitivity of unbiased AMR elements can also be utilized to measure field in two directions [10].

The measured results confirm the fact that in small field region, for which the single-domain structure of the AMR sensor is not broken, simple formulas for crossfield response are valid. 

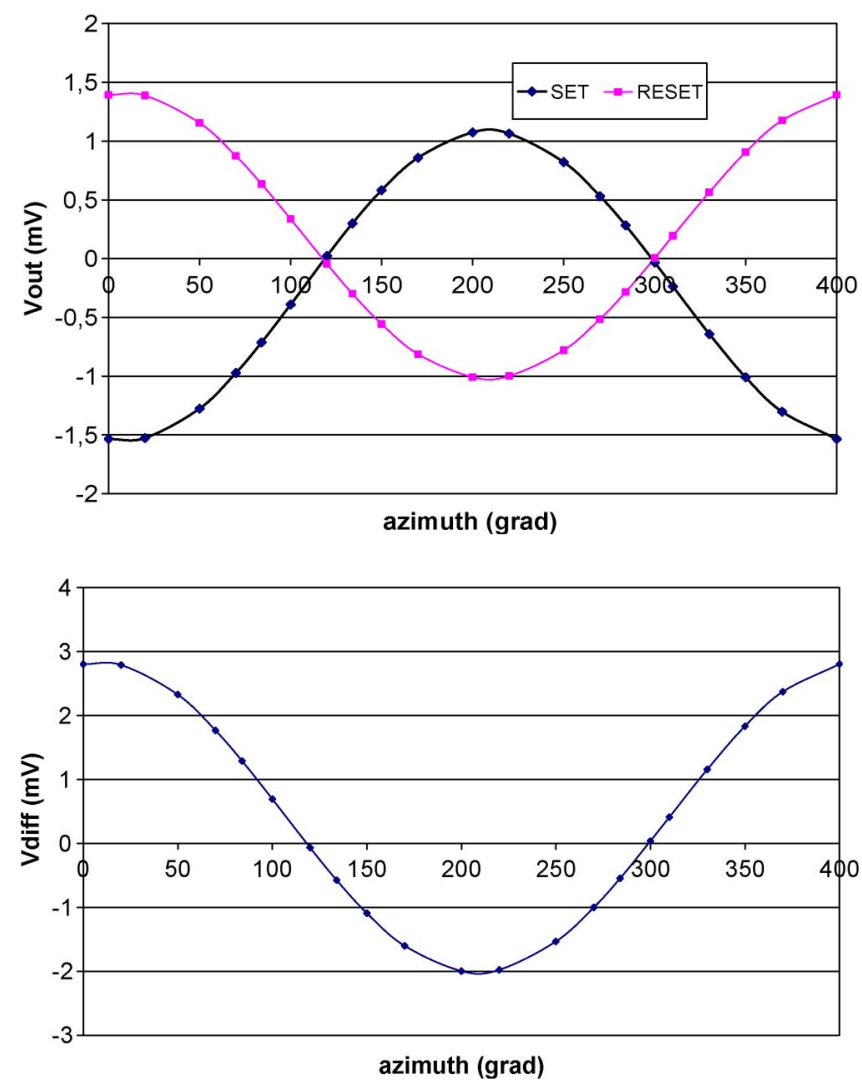

Fig. 3. (a) HMC-1001 sensor's $V_{1}$ (SET) and $V_{2}$ (RESET) outputs for rotation around the crossfield axis $\mathrm{x}$, which was pointing upwards. Notice that for this measurement the sensor was oriented differently than for Figs. 5 and 6. (b) HMC-1001: V diff $=V_{1}-V_{2}$ as a function of rotation around crossfield axis.

We have shown that separate processing of the $\mathrm{V}_{1}$ and $\mathrm{V}_{2}$ voltages instead of simple averaging leads to more efficient correction of the crossfield error. This is easy in digital magnetometers such as [11]. However, if field compensation is possible, such processing is not necessary.

It would be desirable to design more sensitive AMR sensors. One of the possible approaches would be to decrease $H_{0}$. Such sensor would no longer be linear and would have a large crossfield sensitivity, but both these errors can be easily compensated or corrected.

\section{ACKNOWLEDGMENT}

This work was supported by the research program No. MSM6840770015 sponsored by the Ministry of Education, Youth and Sports of the Czech Republic.

\section{REFERENCES}

[1] P. Ripka, Magnetic Sensors and Magnetometers. Boston, MA: Artech, 2001

[2] S. Tumanski, Thin Film Magnetoresistive Sensors. New York: IoP Pub., 2001

[3] B. B. Pant and M. Caruso, Magnetic Sensor Cross-Axis Effect, AN 205 Honeywell.

[4] J. Kubik, J. Vcelak, and P. Ripka, "On cross-axis effect of the anisotropic magnetoresistive sensors," Sens. Actuators A, vol. 129, pp. $15-19,2006$

[5] J. Včelák, P. Ripka, A. Platil, J. Kubík, and P. Kašpar, "Errors of AMR compass and methods of their compensation," Sens. Actuators A, vol. 129 , pp. 53-57, 2006.

[6] P. Ripka and M. Butta, "Origin of the crossfield effect in AMR sensors," presented at the EMSA 2008 Conf., to appear in Sensors Lett..

[7] M. Vopálenský, P. Ripka, and A. Platil, "Precise magnetic sensors," Sens. Actuators A, vol. 106, pp. 38-42, 2003.

[8] F. Primdahl and P. A. Jensen, "Compact spherical coil for fluxgate magnetometer vector feedback," J. Phys. E: Sci. Instrum., vol. 15, pp. 221-226, 1982.

[9] P. Ripka and M. Janosek, "Crossfield sensitivity in AMR sensors and its compensation," in 10th Symp. Magnetoresistive Sensors and Magnetic Systems, Wetzlar, 2009.

[10] B.-Z. Kaplan, E. Paperno, and D. Flunn, "In-plane vector magnetometer employing a single unbiased magnetoresistor," IEEE Trans. Magn., vol. 34, pp. 253-258, 1998.

[11] A. Bertoldi, D. Bassi, and L. Ricci et al., "Magnetoresistive magnetometer with improved bandwidth and response characteristics," Rev. Sci. Instrum., vol. 76, p. 065106, 2005. 